ness of the start is enhanced when, as in the present instance, the book is itself an introduction to a complex subject. But it does not help a beginner if the first chapter is of the nature of a summary which is either superfluous or indigestible. If the beginner in physiology has no previous knowledge of chemistry, physics and biology, he had far better acquire some, because these disciplines provide tools and concepts without which modern physiology cannot be taught or understood. If the student has such background, then this introductory chapter is unnecessary and distracts from the main thread of the book.

But, apart from this quite minor objection-and it is a minor one because certainly few students are likely to use the chapter-the treatment goes along smoothly and competently. The contents of Chapter 2 , which is a brief account of histology, are harmless enough but scarcely necessary if the student has done any general biology. The book seriously settles down to its real business with Chapter 3 , on the blood, tissue fluids, etc., and there is no hitch in the story after that. It is clearly and accurately told, and with a modern point of view ; the figures are numerous and clear ; and stress is laid on aspects of the subject which are of practical importance, such as artificial respiration, shock, blood transfusion, and so on.

One of the difficulties in teaching physiology is that the body works as an integrated whole in which there is complex and continuous interplay between various component parts. Hence the orthodox treatment of the organism by systems is necessarily artificial. The control of function by the central nervous system, by the endocrine organs, and by collaboration between the two is most difficult to bring home at a first presentation. The present authors have tried to bring things together by cross-references as, for example, when dealing with the cardiac nerves by referring to chapters on the centrel nervous system farther on in the book. This is one way out of the difficulty, but in the end the only way seems to be for the student to read a physiology book twice or, better still, if he is intending to tackle the subject seriously, to read and digest such a book as this and then to pass on, with no terrors, to a more advanced book. In giving a clearly written and accurate book of an introductory kind, the authors have rendered real service to the teacher of physiology.

\section{Lovatt Evans}

\section{ANALYSIS OF PERSONALITY}

The Scientific Study of Personality

By Dr. H. J. Eysenck. Pp. xiii+320. (London: Routledge and Kegan Paul, Ltd., 1952.) 30s. net.

HE extensive literature of experimental and
speculative work on the topic of personality has
indicated that the explanations of a person's behaviour
present a particularly complex and intriguing problem.
Both from an empirical and a methodological point
of view, it is one of the most difficult problems ever
to challenge scientific curiosity. The number of
possible causes contributing to behaviour is large and
varied and may involve reference to fields usually
regarded as the province of the economist, historian,
anthropologist, physiologist, neurologist, endocrin-
ologist and geneticist. The problem is rendered even
more difficult by the operation of the large number
of offsetting influences which the individual en-
counters during his life; and the awkward fact for the exponent of correlational techniques is that experienced clinicians can disagree on the classification of the major abnormal states. Again, while reliable class differences may be established between normals, schizophrenics and psychotics in the performance of a particular task, the clinician is expected to understand the context of each patient's difficulties so that his recommendations as to therapy can be effective within their particular environment.

Dr. H. J. Eysenck begins by warning his reader against the 'prestige' value of the words 'science' and 'scientific' which may cloak questionable procedures ; and then indicates that the scientific way of studying personality is to establish principles of classification by the methods of factorial analysis of correlations between the results of a variety of objective tests of behaviour. Although the suggestion of an argumentum ad verecundiam by appeal to the prestige of the methods vindicated in the physical sciences does arise, the operational definition of abnormal mental states by reference to the dimensions established by factorial analysis is lucidly presented. The author and his co-workers have undertaken a considerable amount of statistical labour, and some arresting correlations between test performance and clinical designations are reported. Although the discussion of these findings with reference to earlier work is not always marked by customary scientific humility, comparison with results based upon other populations will be awaited with interest.

The work is a competent exposition of techniques whereby tests indicative of abnormal states may be assessed and refined - an important and useful part of the objective approach in a difficult field. The problem as a whole, however, must surely involve a thorough analysis of environmental conditions associated with abnormal states and some indications as to therapy.

F. V.S.

\section{MODULATION THEORY IN RADIO COMMUNICATION}

\section{Harmonics, Sidebands and Transients in Com- munication Engineering}

As Studied by the Fourier and Laplace Analyses. By C. Louis Cuccia. (McGraw-Hill Electrical and Electronic Engineering Series.) Pp. ix +465 . (London: McGraw-Hill Publishing Co., Ltd., 1952.) 76s. $6 d$.

$7 \mathrm{HE}$ need to increase the speed of radio communication, to provide a wide range of services and to use the available frequency spectrum in the most economical manner has led to the use of complex systems of modulation, and associated mathematical problems of corresponding complexity have arisen. For example, there is often considerable difficulty in the theoretical evaluation of the band-widths required for the transmission of certain types of signal and in the determination of the response of eircuits to applied voltages. The object of Mr. C. L. Cucoia's book is to show how the Fourier series and the Fourier and Laplace transforms can be used for the solution of these and other similar problems. It is essentially a text-book on these functions and is designed particularly for the postgraduate student.

The first four chapters consist of a brief presentation of the Fourier and Laplace methods of analysisperhaps too brief for the reader with no previous knowledge of these methods, but nevertheless a clear 